\title{
Vertebral Osteomyelitis: A Comparison of Associated Outcomes in Early Versus Delayed Surgical Treatment
}

\author{
FRANK A. SEGRETO, BS, ${ }^{1}$ GEORGE A. BEYER, MS, ${ }^{2}$ PRESTON GRIECO, MD ${ }^{3}$ SAMANTHA R. HORN, \\ BA, ${ }^{1}$ COLE A. BORTZ, BA, ${ }^{1}$ CYRUS M. JALAI, BA, ${ }^{1}$ PETER G. PASSIAS, MD,${ }^{1}$ CARL B. PAULINO, MD, ${ }^{3}$ \\ BASSEL G. DIEBO, MD ${ }^{3}$ \\ ${ }^{I}$ Department of Orthopaedics, NYU Langone Medical Center, Hospital for Joint Diseases, New York, New York, ${ }^{2}$ SUNY Downstate College of Medicine, \\ Brooklyn, New York, ${ }^{3}$ Department of Orthopaedic Surgery, SUNY Downstate, Brooklyn, New York
}

\begin{abstract}
Background: The recommended timing of surgical intervention for vertebral osteomyelitis (VO) is controversial; however, most studies are not sufficiently powered. Our goal was to investigate the associated effects of delaying surgery in VO patients on in-hospital complications, neurologic deficits, and mortality.

Methods: Retrospective review of the National Inpatient Sample. Patients who underwent surgery for VO from 1998 to 2013 were identified using codes from the International Classification of Disease, Ninth Revision, Clinical Modification. Patients were stratified into groups based on incremental delay of surgery: 0-day delay (same-day surgery), 1-day delay, 2-day delay, 3- to 6-day delay, 7- to 14-day delay, and 14- to 30-day delay. Univariate analysis compared demographics and outcomes between groups. Multivariate logistic regression models calculated independent predictors of any complication, mortality, and neurologic deficits. A 0-day delay was the reference group.

Results: A total of 34465 patients were identified. Delayed groups were older (same day: 53.5 vs. 7-14-day delay: 61.1) and had a higher Deyo-Charlson score (same day: 0.4901 vs. 14-30-day delay: 1.66), length of stay (same day: 4.2 vs. 14-30-day delay: 34.04 days), and total charges (same day: $\$ 63,390.78$ vs. 14-30-day delay: $\$ 245,752.4$ ), all $P<.001$. Delayed groups had higher surgical combined-approach rates (same day: $9.1 \%$ vs. 14 -30-day delay: $31.5 \%$ ) and lower anterior-approach rates (same day: $42.4 \%$ vs. $14-30$-day delay: $24.2 \%$ ). Delayed groups had increased mortality and complication rates. Regressions showed delayed groups as the strongest independent indicators of any complication (14-30-day delay: odds ratio [OR] 3.384), mortality (14-30-day delay: OR 10.658), and neurologic deficits (14-30-day delay: OR 3.464), all $P<.001$.

Conclusion: VO patients who operate within 24 hours of admission are more likely to have desirable outcomes. Patients with delayed surgery had a significantly increased risk of developing any complication, mortality, and discharging with neurologic deficits.

Level of Evidence: III.

Clinical Relevance: Medically fit patients may benefit from earlier surgical management in order to reduce risk of postoperative complications, improve outcomes, and reduce overall hospital costs.

Other and Special Categories
\end{abstract}

Keywords: vertebral osteomyelitis, surgical delay, spinal fusion, spondylodiscitis

\section{INTRODUCTION}

Vertebral osteomyelitis (VO) is a rare spine infection often developing from open spinal trauma, infections in adjacent anatomic structures, or hematogenous spread of bacteria or as a postoperative complication to spinal surgery. ${ }^{1-4}$ Although VO remains uncommon, with yearly rates of 5 to 20 cases per million in the United States, its incidence is increasing. ${ }^{3,5-7}$ This is believed to be attributed to the increase of patients with advanced age, diabetes mellitus, chronic renal or liver disease, intravenous drug use, HIV infection, chronic corticosteroid use, chemotherapy, and severe trauma-all predisposing factors to VO. ${ }^{8,9}$ Since 1974, the incidence has nearly doubled from 0.2 to 2 cases per 10000 hospital admissions to 2 to 3 cases per 10000 hospital admissions, ${ }^{10-12}$ with a 20 -fold-higher incidence in older patients. ${ }^{13}$ If left untreated, VO can lead to irreversible spinal cord injury, deformity, neurologic deficits, septicemia, and mortality (mortality rates ranges $4 \%-29 \%)^{4,14} \mathrm{VO}$ is traditionally treated conservatively with antimicrobial therapies, but up to $40 \%$ to $50 \%$ of $\mathrm{VO}$ patients suffering will eventually require surgical intervention. ${ }^{4,15}$ 
VO surgical intervention is indicated when patients express progressive loss of motor and/or neurological functions, cauda equine syndrome, progressive deformities, spinal instability, abscess formation, and delayed diagnosis. Failure of conservative treatment categorized as persistent pain, residual neurologic deficits, and systemic inflammation/infection also warrants surgical intervention. While clinicians have agreed on surgical indications for VO, a persistent area of controversy is the optimal timing of surgery that most benefits the patient. ${ }^{14}$ Prior studies report conflicting findings: Ghobrial et $\mathrm{al}^{16}$ in 2014 and Connor et $\mathrm{al}^{18}$ in 2013 reported a relative advantage to earlier surgical intervention with regard to discharge neurological status, while Adogwa et $\mathrm{al}^{17}$ in 2014 and Karikari et $\mathrm{al}^{10}$ in 2009 reported no statistical benefit from early surgical intervention on postoperative outcomes. While these studies present relative insight into the timing of surgical intervention, they are limited by small sample sizes (82-104 patients), minimal subgroup analysis, and institutional bias.

This study evaluates the outcomes of early $(<24$ hours) versus delayed surgical treatment of VO using a large nationwide inpatient database. Our study also aimed to investigate the demographics, comorbidities, socioeconomics, and prevalence of multiple complications associated with variant surgical timing of the VO patient population.

\section{MATERIALS AND METHODS}

\section{Data Source}

This is a retrospective review of the National Inpatient Sample (NIS) from 1998 to 2013. The NIS is the largest available all-payer database for US inpatient care and includes data approximating 8 million discharges from 1000 hospitals annually. Forty-five states are represented in the NIS, with approximately a $20 \%$ stratified random sample of all US community hospitals. The Health Care Cost and Utilization Project provides support for the NIS, with further support coming from federal, state, and industrial partnerships. The NIS presents clinical, demographic, diagnosis, and procedural data elements in the International Classification of Disease, Ninth Revision, Clinical Modification (ICD-9-CM) format. More information can be found at http://hcup-us.ahrq.gov/db/nation/nis/ NIS_Introduction_2012.jsp. This study was deemed exempt by our institutional review board due to the deidentified nature of the data.

\section{Inclusion Criteria}

Patients of all ages who underwent surgical treatment for VO from 1998 to 2013 were identified. ICD-9 codes identified patients who underwent surgery for VO. Patients were then stratified based on procedural delay into 6 groups: 0 days (same day as admission surgery), 1 day, 2 days, 3 to 6 days, 7 to 14 days, and 14 to 30 days. Patients with delays $>30$ days, representing less than $0.1 \%$ of the total subset, were excluded due to wide variability in surgical delays often related to the presence of significant comorbidities.

\section{Data Collection}

Demographics, including age, gender, race, length of hospital stay, total charges, comorbidity status, and insurance type, were analyzed. The Deyo-Charlson Index, a modification by Deyo et $\mathrm{al}^{19}$ of the Charlson comorbidity severity index for the utilization of ICD-9-CM diagnostic and procedural codes, was used to describe comorbidity severity within our cohorts. The comorbidities assessed in this study and included within the index were myocardial infarction, congestive heart failure, pulmonary vascular disease, stroke, dementia, chronic pulmonary disease, rheumatological conditions, peptic ulcer disease, liver disease, diabetes, diabetes with complications, renal disease, cancer, severe liver disease, metastasis, and AIDS. Subsets of VO surgical patients were investigated by the method of surgical approach (anterior, posterior, and combined anterior/posterior) and the spinal level (cervical, thoracic, lumbosacral) of the operation. Perioperative surgical and medical complications were identified and further classified into the following subsets: the top 3 most common complications, rate of any complications, and mortality. All elements were identified using ICD-9 codes (Appendix A).

\section{Neurologic Index}

A neurologic index was created using the design of the American Spinal Injury Association (ASIA) impairment scale as a reference. Numerical scores between 0 and 2 were assigned to patients with corresponding neurological deficits. A score of 0 was assigned to patients without any neurologic 


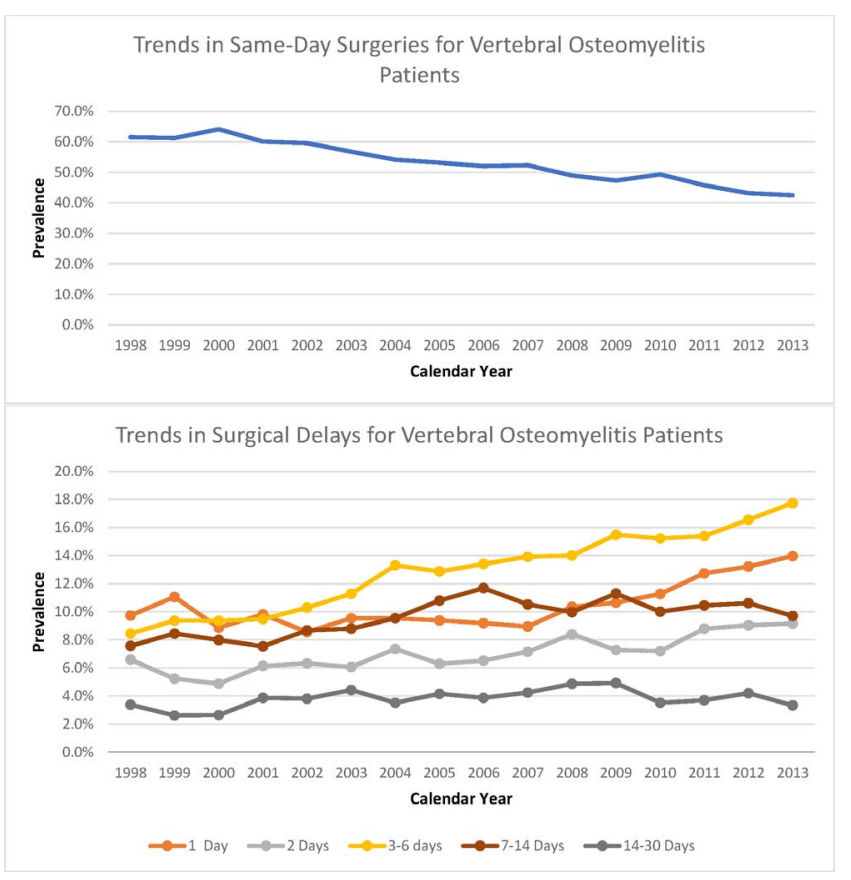

Figure 1. Trends in same-day surgeries and surgical delays for vertebral osteomyelitis (VO) patients from 1998 to 2013. The $y$-axis percentages represent the prevalence of patients in a specified surgical group in relation to the total amount of surgical VO patients for each corresponding $x$-axis year.

deficits (ASIA grade E), a score of 1 to patients with incomplete neurologic deficit (ASIA grades B-D), and a score of 2 to patients with complete neurologic deficit (ASIA grade A). Incomplete neurologic deficits include paraplegia (upper or lower), radiculopathy, myelopathy, cauda equina syndrome, and spondylosis with compression. Complete neurologic deficits include quadriplegia and complete paralysis.

\section{Study Design and Statistical Analysis}

Descriptive analysis was used to analyze the demographics and comorbidities. Trends of sameday surgeries and delayed surgeries between 1998 and 2013 were plotted. Varying surgical groups were compared via univariate analysis. Independentsample $t$ tests and 1-way analysis of variance elucidated significant variation in age, Deyo-Charlson Index, total hospital charges, and length of stay. Chi-square tests evaluated variations in patient distribution between the surgical delay groups with regard to demographic data (insurance, race, gender) as well as surgical information (complication type, complication rates, spinal level, surgical approach, neurologic index). Three binary multiple logistic forward stepwise regression models were generated to identify independent predictors of any complication, mortality, and neurologic index $>0$. Models controlled for age, gender, Deyo-Charlson score, and surgical approach. The level of significance was set to $P<.05$. Statistical analyses were performed using IBM SPSS Statistics 23.

\section{RESULTS \\ Trends of Surgical Timing}

Since 1998, the incidence of patients undergoing surgical treatment has drastically increased. In 1998, 1243 patients underwent surgical intervention. Since then, there has been an incremental increase to 3155 patients undergoing surgical treatment in 2013. The prevalence of VO patients undergoing same-dayadmission surgeries decreased from $61.5 \%$ in 1998 to $42.5 \%$ in 2013 , while the prevalence of $\mathrm{VO}$ patients undergoing surgical delays has increased. In 1998, patients with 3 to 6 days of surgical delay had a rate of $8.4 \%$, which increased to $17.7 \%$ in 2013 (Figure 1). Of all years examined, 2013 showed the highest prevalence of patients undergoing surgery with a 1-day delay $(14 \%)$, 2-day delay $(9.2 \%)$, and 3- to 6-day delay (17.7\%) (Figure 1).

\section{General Analysis Between Surgical Delay Groups}

A total of 34465 patients fulfilled the inclusion criteria for this study. Patients undergoing same-day surgery had a mean age of 53.48 years. Mean age increased incrementally in successive surgical delay groups (7-14 days: 61.05 years), apart from patients with 14- to 30-day delays (60.22 years). Same-daysurgery patients had a significantly lower DeyoCharlson Index (0.49) than those with surgical delays as short as 1 day (1.08). Further, the DeyoCharlson Index increased in every successive surgical delay group. The proportion of black and Hispanic patients increased in successive surgical delay groups. The prevalence of those with Medicare (same day: $29.3 \%$ vs. $14-30$-day delay: $51.2 \%$ ) and Medicaid (same day: $6.1 \%$ vs. $14-30$-day delay: $16.2 \%$ ) increased in successive surgical delay groups. The variations between surgical groups for all demographic variables tested were statistically significant $(P<.001)$ (Table 1$)$.

The most prevalent comorbidities identified in same-day-surgery patients were diabetes $(12.9 \%)$, chronic pulmonary disease $(11.9 \%)$, and myocardial infarction $(2.2 \%)$. All investigated comorbidities showed significant increases in prevalence with successive surgical delay $(P<.001)$. Comorbidities 
Table 1. Demographics of vertebral osteomyelitis surgical patients with varied surgical delays. Bolded cells with an asterisk represent a significance of $P<.05$.

\begin{tabular}{|c|c|c|c|c|c|c|c|}
\hline & Same Day & $1 \mathrm{~d}$ & $2 d$ & $3-6 d$ & $7-14 \mathrm{~d}$ & $14-30 \mathrm{~d}$ & $P$ Value \\
\hline Sample size & 17693 & 3725 & 2536 & 5780 & 3391 & 1340 & \\
\hline Mean age & 53.48 & 57.95 & 58.98 & 60.23 & 61.05 & 60.22 & $<.001 *$ \\
\hline Length of stay & 4.20 & 10.10 & 11.19 & 13.95 & 20.64 & 34.04 & $<.001 *$ \\
\hline Total charges & 63390.78 & 84995.54 & 95425.43 & 111013.7 & 155282.86 & 245752.4 & $<.001 \%$ \\
\hline Deyo-Charlson Index & 0.49 & 1.08 & 1.19 & 1.39 & 1.57 & 1.67 & $<.001^{*}$ \\
\hline \multicolumn{8}{|l|}{ Gender } \\
\hline Male & $51.20 \%$ & $59.80 \%$ & $58.30 \%$ & $57.90 \%$ & $56.70 \%$ & $56.70 \%$ & \multirow[t]{2}{*}{$<.001 *$} \\
\hline Female & $48.80 \%$ & $40.20 \%$ & $41.70 \%$ & $42.10 \%$ & $43.30 \%$ & $43.30 \%$ & \\
\hline \multicolumn{8}{|l|}{ Race } \\
\hline White & $79.10 \%$ & $75.30 \%$ & $71.80 \%$ & $69.0 \%$ & $66.00 \%$ & $61.40 \%$ & \multirow[t]{4}{*}{$<.001 *$} \\
\hline Black & $8.10 \%$ & $11.20 \%$ & $12.90 \%$ & $15.3 \%$ & $17.00 \%$ & $19.50 \%$ & \\
\hline Hispanic & $8.70 \%$ & $9.00 \%$ & $9.80 \%$ & $10.3 \%$ & $10.70 \%$ & $12.80 \%$ & \\
\hline Other & $4.20 \%$ & $4.50 \%$ & $5.50 \%$ & $5.4 \%$ & $6.20 \%$ & $6.20 \%$ & \\
\hline \multicolumn{8}{|l|}{ Insurance } \\
\hline Medicare & $29.3 \%$ & $42.5 \%$ & $46.2 \%$ & $51.8 \%$ & $55.5 \%$ & $51.2 \%$ & \multirow[t]{6}{*}{$<.001 *$} \\
\hline Medicaid & $6.1 \%$ & $11.1 \%$ & $12.2 \%$ & $12.6 \%$ & $12.4 \%$ & $16.2 \%$ & \\
\hline Private insurance & $46.6 \%$ & $33.3 \%$ & $30.1 \%$ & $25.2 \%$ & $22.6 \%$ & $22.1 \%$ & \\
\hline Self-Pay & $1.6 \%$ & $5.4 \%$ & $5.1 \%$ & $4.9 \%$ & $4.4 \%$ & $5.2 \%$ & \\
\hline No charge & $0.2 \%$ & $0.6 \%$ & $0.8 \%$ & $0.6 \%$ & $0.7 \%$ & $0.8 \%$ & \\
\hline Other & $16.2 \%$ & $7.1 \%$ & $5.6 \%$ & $4.9 \%$ & $4.6 \%$ & $4.5 \%$ & \\
\hline
\end{tabular}

that were most prevalent in the 14- to 30-day-delay group included congestive heart failure (14.8\%), renal disease $(14.8 \%)$, diabetes with complications $(7.9 \%)$, cancer $(6.4 \%)$, and stroke $(4.0 \%)$ (Table 2$)$.

\section{In-Hospital Perioperative Outcomes of Patients With Varying Surgical Delays}

Patients in all groups were more likely to undergo posterior-only approaches (range: $41.7 \%-48.4 \%$ ). In comparison to surgically delayed patients, sameday-surgery patients had higher anterior-only approach rates (same day: $42 \%$ vs. $7-14$-day delay: $24.6 \%, P<.05)$. Surgically delayed patients were more likely to undergo combined approaches (same day: $9.1 \%$ vs. $14-30$-day delay: $31.5 \%, P<.05$ ). The lumbosacral region had the highest operative rates among all groups (range: 41.6\%-62.8\%). Cervical procedures were most prevalent less delayed groups (same day: $32.6 \%, P<.001$ ), while thoracic procedures were most prevalent in surgically delayed patients (14-30-day delay: $34.6 \%$, $P<$.001) (Table 3).

Anemia was the most common complication in same-day-surgery patients $(6.1 \%)$. Infection was the most common complication in patients with 1-day delay $(7.9 \%)$. Subsequently, each successive surgical delay group had sepsis as the most common complication (range: $8.2 \%-19.3 \%$ ). Mortality in same-day-surgery patients was $0.3 \%$, with successive surgical delay groups experiencing increased mortality rates, peaking at 5.5\% (14-30-day delay, $P<.001)$. The occurrence of any complication progressively increased from $16.7 \%$ (same day) to 42.5\% (14-30-day delay, $P<.001)$. At discharge, same-day-surgery patients had a $7.2 \%$ rate of incomplete neurological deficits. Surgically delayed

Table 2. Comorbidities of patients with varying surgical delays. Bolded cells with an asterisk represent a significance of $P<.05$.

\begin{tabular}{|c|c|c|c|c|c|c|c|}
\hline & Same Day, \% & $1 \mathrm{~d}, \%$ & $2 \mathrm{~d}, \%$ & $3-6 \mathrm{~d}, \%$ & $7-14 \mathrm{~d}, \%$ & $14-30 \mathrm{~d}, \%$ & $P$ Value \\
\hline Diabetes & 12.90 & 17.60 & 18.10 & 19.30 & 19.20 & 18.90 & $<.001 *$ \\
\hline Chronic pulmonary disease & 11.90 & 12.10 & 12.30 & 14.50 & 14.90 & 12.40 & $<.001 *$ \\
\hline Myocardial infarction & 2.20 & 2.90 & 2.20 & 3.10 & 3.50 & 2.60 & $<.001 *$ \\
\hline Rheumatological conditions & 2.00 & 3.00 & 3.20 & 3.30 & 2.90 & 3.10 & $<.001 *$ \\
\hline Renal disease & 1.90 & 7.00 & 9.40 & 11.90 & 14.20 & 14.80 & $<.001 *$ \\
\hline Congestive heart failure & 1.80 & 5.30 & 8.20 & 9.40 & 12.90 & 14.80 & $<.001 *$ \\
\hline Diabetes with complications & 1.30 & 3.90 & 5.60 & 6.10 & 7.60 & 7.90 & $<.001 *$ \\
\hline Pulmonary vascular disease & 1.20 & 2.10 & 3.40 & 4.30 & 3.70 & 3.20 & $<.001 *$ \\
\hline Cancer & 1.10 & 4.20 & 5.00 & 4.90 & 6.20 & 6.40 & $<.001 *$ \\
\hline Any stroke & 0.80 & 1.90 & 1.40 & 2.50 & 3.40 & 4.00 & $<.001 *$ \\
\hline Peptic ulcer disease & 0.60 & 0.90 & 1.30 & 1.00 & 1.40 & 2.30 & $<.001 *$ \\
\hline Liver disease & 0.40 & 2.50 & 2.10 & 2.50 & 2.50 & 2.80 & $<.001 *$ \\
\hline Metastasis & 0.30 & 1.60 & 1.70 & 2.00 & 1.70 & 2.80 & $<.001 *$ \\
\hline AIDS & 0.20 & 0.90 & 0.80 & 1.10 & 1.40 & 1.00 & $<.001 *$ \\
\hline Severe liver disease & 0.10 & 0.50 & 0.80 & 1.00 & 1.20 & 2.00 & $<.001 *$ \\
\hline Dementia & 0.00 & 0.20 & 0.00 & 0.30 & 0.60 & 0.60 & $<.001 *$ \\
\hline
\end{tabular}


Table 3. Overview of surgical approach in patients with varied surgical delays. Bolded cells with an asterisk represent a significance of $P<.05$.

\begin{tabular}{|c|c|c|c|c|c|c|c|}
\hline & Same Day, \% & $1 \mathrm{~d}, \%$ & $2 \mathrm{~d}, \%$ & $3-6 \mathrm{~d}, \%$ & $7-14 \mathrm{~d}, \%$ & $14-30 \mathrm{~d}, \%$ & $P$ Value \\
\hline \multicolumn{8}{|c|}{ Surgical approach } \\
\hline Anterior & 42.4 & 36.5 & 33.8 & 33.3 & 24.6 & 24.2 & \multirow[t]{3}{*}{$<.001 *$} \\
\hline Posterior & 48.4 & 47.6 & 41.7 & 44.1 & 46.5 & 44.3 & \\
\hline Combined & 9.1 & 15.9 & 24.4 & 22.6 & 28.9 & 31.5 & \\
\hline \multicolumn{8}{|l|}{ Surgical location } \\
\hline Cervical & 32.6 & 33.6 & 29.1 & 27.8 & 22.4 & 20.1 & \multirow[t]{3}{*}{$<.001 *$} \\
\hline Thoracic & 4.6 & 24.8 & 28.3 & 27.9 & 33.9 & 34.6 & \\
\hline Lumbosacral & 62.8 & 41.6 & 42.6 & 44.3 & 43.7 & 45.3 & \\
\hline
\end{tabular}

patients showed significantly higher rates of incomplete neurologic deficits (range: $11.5 \%-14.7 \%$, $P<.001)$ at discharge. Rates of complete neurologic deficit also showed significant variation among groups, with a higher prevalence in delayed groups (14-30-day delay: $0.4 \%, P<.05$ ) (Table 4).

Regression models revealed that all surgical delay groups $\geq 1$ day were the strongest predictors of complications, mortality, and a neurologic index $>0$. The most significant independent predictor of the occurrence of any complication was a surgical delay of 14 to 30 days $(\mathrm{B}=1.219$, odds ratio $[\mathrm{OR}]=$ 3.384 [2.830-4.046], $P<.001)$, while a surgical delay of 7 to 14 days was the second most significant independent predictor of any complication $(\mathrm{B}=$ 0.984$, OR $=2.675[2.346-3.051], P<.001) . \mathrm{A}$ combined surgical approach further predicted complication rate $(\mathrm{B}=0.973, \mathrm{OR}=2.647$ [2.360-2.969], $P<.001)$. After surgical delay groups, the DeyoCharlson Index was the next most significant predictor of mortality $(\mathrm{B}=0.232$, OR $=1.261$ [1.179-1.348], $P<.001)$ and neurologic index $>0$ $(\mathrm{B}=0.493, \mathrm{OR}=1.637[1.566-1.711], P<.001)$ (Table 5).

\section{DISCUSSION}

Trends over the past 2 decades have shown a progressive increase of incidence in VO. ${ }^{3,5-7}$ Elderly patients are particularly at risk, ${ }^{13}$ and with the US annual increase in life expectancy, ${ }^{20} \mathrm{VO}$ has become an increasingly relevant concern for health care providers. VO's high operative and mortality rates ${ }^{4,14,15}$ pose a need for more information concerning surgical treatment and perioperative outcomes. Currently, the recommended timing of surgical intervention for VO patients is very controversial.

This study found that VO patients who underwent surgery after 24 hours of admission had higher chances of mortality, being discharged with an impaired neurologic status, and having any type of perioperative complication. Specifically, there were higher rates of sepsis, anemia, and recurrent infection in surgically delayed patients. These patients were also more likely to have a combined anterior-posterior surgery and less likely to undergo an anterior approach. Surgeries were more likely to be performed at the thoracic than cervical level in surgically delayed patients. Demographically, our results indicate that surgically delayed VO patients were more likely to be older, male, black or Hispanic, and on Medicare or Medicaid and to have increased total hospital charges. Patients experiencing surgical delays predictably had higher Deyo-Charlson scores and increased comorbidity rates. While the Deyo-Charlson score was shown to be a significant predictor of adverse outcomes, controlled regression models showed that surgical delay of any degree most greatly predicted mortality, impaired neurologic status, and the chance of developing any complication. This reduces the confounding nature of the association between the

Table 4. Prevalence of most common complications and neurologic deficits in groups with varied surgical delays. Bolded cells with an asterisk represent a significance of $P<.05$.

\begin{tabular}{|c|c|c|c|c|c|c|c|}
\hline Complications & Same Day, \% & $1 \mathrm{~d}, \%$ & $2 \mathrm{~d}, \%$ & $3-6 \mathrm{~d}, \%$ & $7-14 \mathrm{~d}, \%$ & $14-30 \mathrm{~d}, \%$ & $P$ Value \\
\hline Most common & Anemia (6.1) & Infection (7.9) & Sepsis (8.2) & Sepsis (10.6) & Sepsis (14.5) & Sepsis (19.3) & - \\
\hline Second most common & Device (3.3) & Sepsis $(7.1)$ & Anemia (6.1) & Anemia (6.8) & Anemia (8.4) & Anemia (10.5) & - \\
\hline Third most common & Infection (2.0) & Anemia (6.9) & Infection (6.0) & Infection (5.4) & Infection (5.4) & Infection (8.5) & - \\
\hline Mortality & 0.3 & 1.7 & 2.15 & 2.3 & 3.1 & 5.5 & $<.001 *$ \\
\hline Rate of any complication & 16.7 & 25.6 & 23.8 & 27.1 & 33.2 & 42.5 & $<.001 *$ \\
\hline \multicolumn{8}{|l|}{ Neurologic index } \\
\hline Incomplete neurologic deficit & 7.2 & 11.9 & 11.5 & 11.7 & 12.9 & 14.7 & $<.001 *$ \\
\hline Complete neurologic deficit & 0.0 & 0.0 & 0.1 & 0.0 & 0.1 & 0.4 & $<.001 *$ \\
\hline
\end{tabular}


Table 5. Demographic information, surgical delay, and characteristics of surgery as independent predictors of any complication, mortality, and neurologic index. "-" indicates that the variable failed to enter the regression model. Bolded cells with an asterisk represent a significance of $P<.05$.

\begin{tabular}{|c|c|c|c|}
\hline Predictor & B-Coefficient & Odds Ratio (95\% Confidence Interval) & $P$ Value \\
\hline \multicolumn{4}{|l|}{ Any complication } \\
\hline Age & 0.017 & $1.017(1.014-1.020)$ & $<.001 *$ \\
\hline Gender & - & - & - \\
\hline Deyo-Charlson Index & 0.030 & $1.031(1.002-1.061)$ & $.038 *$ \\
\hline 1-d delay & 0.802 & $2.230(1.935-2.570)$ & $<.001 *$ \\
\hline 2-d delay & 0.637 & $1.891(1.591-2.247)$ & $<.001 \%$ \\
\hline 3- to 6-d delay & 0.889 & $2.433(2.165-2.735)$ & $<.001 *$ \\
\hline 1-wk to 14 -d delay & 0.984 & $2.675(2.346-3.051)$ & $<.001 *$ \\
\hline 14-d to 1-mo delay & 1.219 & $3.384(2.830-4.046)$ & $<.001 *$ \\
\hline Year of surgery & - & - & - \\
\hline Posterior surgical approach & 0.537 & $1.710(1.561-1.874)$ & $<.001 *$ \\
\hline Anterior surgical approach & 0.973 & $2.647(2.360-2.969)$ & $<.001 *$ \\
\hline Surgical invasiveness & 0.051 & $1.052(1.041-1.065)$ & $<.001 *$ \\
\hline \multicolumn{4}{|l|}{ Mortality } \\
\hline Age & 0.051 & $1.053(1.040-1.066)$ & $<.001 *$ \\
\hline Gender & -0.327 & $0.721(0.525-0.990)$ & $.043 *$ \\
\hline Deyo Index & 0.232 & $1.261(1.179-1.348)$ & $<.001 *$ \\
\hline $1-d$ delay & 1.730 & $5.643(3.138-10.146)$ & $<.001 *$ \\
\hline 2-d delay & 1.580 & $4.854(2.447-9.626)$ & $<.001 *$ \\
\hline 3- to 6-d delay & 1.945 & $6.994(4.256-11.493)$ & $<.001 *$ \\
\hline 1 -wk to 14 -d delay & 2.031 & $7.623(4.538-12.807)$ & $<.001 *$ \\
\hline 14-d to 1 -mo delay & 2.366 & $10.658(6.020-18.868)$ & $<.001 *$ \\
\hline Year of surgery & -0.76 & $0.927(0.896-0.959)$ & $<.001 *$ \\
\hline Posterior surgical approach & -0.625 & $0.535(0.377-0.760)$ & $<.001 \%$ \\
\hline Anterior surgical approach & -0.274 & $0.760(0.503-1.151)$ & .195 \\
\hline Surgical invasiveness & - & - & - \\
\hline \multicolumn{4}{|l|}{ Neurologic index $>0$} \\
\hline Age & -0.12 & $0.989(0.981-0.996)$ & $.002 *$ \\
\hline Gender & -0.290 & $0.748(0.611-0.916)$ & $.005 *$ \\
\hline Deyo Index & 0.493 & $1.637(1.566-1.711)$ & $<.001 *$ \\
\hline 1-d delay & 1.573 & $4.823(3.536-6.578)$ & $<.001 \%$ \\
\hline $2-d$ delay & 0.960 & $2.611(1.718-3.966)$ & $<.001 *$ \\
\hline 3- to 6-d delay & 1.118 & $3.060(2.271-4.121)$ & $<.001 *$ \\
\hline 1 -wk to 14 -d delay & 1.295 & $3.652(2.685-4.969)$ & $<.001 *$ \\
\hline 14-d to $1-$ mo delay & 1.242 & $3.464(2.357-5.086)$ & $<.001 *$ \\
\hline Year of surgery & - & - & - \\
\hline Posterior surgical approach & 0.368 & $1.445(1.102-1.895)$ & $.008 *$ \\
\hline Anterior surgical approach & 0.344 & $1.410(1.034-1.923)$ & $.030 \%$ \\
\hline Surgical invasiveness & 0.123 & $1.131(1.105-1.158)$ & $<.001 *$ \\
\hline
\end{tabular}

Deyo-Charlson score and surgical delay, further justifying our findings.

This study is the first to review a large VO cohort, derived from a nationwide database, to delineate associated effects of surgical delay. Comparatively, the majority of papers investigating surgical indications and treatment outcomes for VO patients have cohorts ranging between 10 and 300 patients. Previous studies by Miller et al, ${ }^{21}$ Adogwa et al, ${ }^{17}$ Kothari et al, ${ }^{22}$ Arnold et $\mathrm{al}^{23}$ and Mavrogenis et $\mathrm{al}^{24}$ all had cohort sizes of 50, 82, 16, 94, and 13 patients, respectively. Of the few studies that did use large database cohorts, such as Akiyama et $\mathrm{al}^{25}(\mathrm{n}=$ 7118), none investigated the effects of delayed surgical treatment. Previous studies focusing on the timing of surgical intervention, such as Ghobrial et al, ${ }^{16}$ reported that surgical evacuation of abscess within 24 hours of admission showed a relative advantage to discharge neurologic grade in com- parison to delayed surgical patients. The findings of Connor et $\mathrm{al}^{18}$ strongly support immediate surgical decompression, combined with appropriately tailored antibiotic therapy for optimal neurologic outcomes. Alternatively, studies such as those by Karikari et $\mathrm{al}^{10}$ and Adogwa et $\mathrm{al}^{17}$ reported no benefit to early surgical intervention for abscess removal.

Despite the controversial nature of our findings, the mortality rates presented in our cohort (which range between $0.3 \%$ and $5.5 \%$ ) seem to be relatively more consistent than the majority of reports in the literature. Akiyama et $\mathrm{al}^{25}$ McHenry et al, ${ }^{26}$ Grammatico et al, ${ }^{7}$ Hutchinson et al, ${ }^{27}$ and Mylona et $\mathrm{al}^{1}$ reported in-hospital mortality rates of $6 \%$, $11 \%, 3 \%, 27 \%$, and $6 \%$, respectively. The slightly lower rates observed in our cohort may be an implication of our VO patient population being limited to surgical candidates only. Miller et $\mathrm{al}^{21}$ (n 
$=50$ ) reported a mortality rate of $2 \%$ within the 30 day postoperative period, while Matsui et $\mathrm{al}^{28}(\mathrm{n}=$ 38) reported a postoperative mortality rate of $0 \%$.

For the majority of patients, anterior vertebral elements are most commonly involved with infection, while posterior elements are affected mainly in patients with advanced infection. In general, most authors advocate anterior procedures for extensive disc and vertebral body debridement in that early surgical patients, who may be in better health and of higher bone quality, may not need the additional stabilization provided by the posterior approach. ${ }^{5}$ Arnold et $\mathrm{al}^{23}$ observed that in patients with successful surgical treatments, $32 \%$ had an anterior approach, 24\% had a posterior approach, and 44\% had a combined approach. Pourtaheri et $\mathrm{al}^{29}$ observed that in patients with instrumented surgical treatment, $58 \%$ had an anterior approach, $13 \%$ had a posterior approach, and $29 \%$ had a combined approach. While our rates for the anterior approach seem to align with previous literary reports, our high posterior-approach rates seem to be discrepant. This may be explained by the belief that the posterior approach is associated with fewer overall postoperative complications ${ }^{30}$ regardless of higher wound infection rates. ${ }^{31}$ Cohort size differences and/or surgeon preference of smaller studies may also be to blame. Our data also showed that combined surgical approach rates incrementally increased with further delay of surgery. This may be attributed to the complexity of the procedure that typically requires a 2-stage operation with extensive planning. ${ }^{14}$

We also found a higher prevalence of surgically delayed VO patients with Medicare and Medicaid insurance. Previous studies have indicated that patients on Medicaid have a hindered access to health care, ${ }^{32}$ longer and more costly hospitalizations, ${ }^{33}$ and higher rates of delayed cancer diagnosis, resulting in higher mortality rates, ${ }^{34,35}$ and are less likely to receive cancer-directed surgery ${ }^{36}$ in comparison to privately insured patients. If these trends hold true for patients with VO, delay of diagnosis and health care providers' reluctance to provide surgical care is extremely detrimental to a patient suffering from VO. Timely diagnoses and earlier surgical care may reduce inpatient length of stay, decrease total hospital costs, lower mortality rates, and improve a patient's overall quality of care. Effectively, Furlan et $\mathrm{al}^{37}$ provided an economic cost-utility analysis for patients undergo- ing early versus delayed surgical spinal cord decompression. They found that early surgical decompression was more cost effective and resulted in improved patient's quality of life in comparison to delayed surgical decompression.

Information is needed with regard to the causative factors that are at play in patients receiving surgical treatment for VO. While the NIS is one of the largest health care databases available, there are confounding variables that are neither identifiable nor controlled for. For example, patient frailty may play a significant role in surgical decision making and influence the decision to delay a surgical procedure. While frailty indexes have been developed for other large databases, such as the National Surgical Quality Improvement Program, ${ }^{38}$ to the best of our knowledge, no previous frailty measures have been validated for use on the NIS, most likely due to a lack of specificity among clinical metrics. The use of ICD-9 codes also limits our ability to determine causation of outcomes. Further, the NIS includes only inpatient data, without outpatient claims, follow-up, and patient-reported outcomes. This is an important limitation to consider, specifically with the usage of the Deyo-Charlson Index. The Deyo-Charlson Index, which includes only data from the inpatient setting, may not include important comorbidities that are recorded on outpatient claims, creating a misrepresentation of a patient's true comorbidity severity. ${ }^{39}$ The NIS database also lacks specific information regarding the patient's clinical course and operation. Finally, this paper does not detail the varying organisms responsible for infection, which may have a profound effect on outcomes. Further information on patient history, clinical course, and follow-up outcomes is needed to create a complete understanding with regard to the management of VO.

\section{CONCLUSION}

This study explores the associated effects of surgical delays in patients with VO. Using a nationwide database, the prevalence of various outcomes in VO patients who underwent early versus delayed surgical procedures was compared. Patients with surgical delays were more likely to have higher mortality rates, develop any complication, or discharge with neurologic deficits. Surgically delayed patients were also more likely to have a greater length of stay and higher total hospital charges. Medically fit patients may benefit from 
earlier surgical management in order to reduce risk of postoperative complications, improve outcomes, and reduce overall hospital costs.

\section{REFERENCES}

1. Mylona E, Samarkos M, Kakalou E, Fanourgiakis P, Skoutelis A. Pyogenic vertebral osteomyelitis: a systematic review of clinical characteristics. Semin Arthritis Rheum. 2009;39(1):10-17.

2. Schimmer RC, Jeanneret C, Nunley PD, Jeanneret B. Osteomyelitis of the cervical spine: a potentially dramatic disease. J Spinal Disord Tech. 2002;15(2):110-117.

3. Jaramillo-de la Torre JJ, Bohinski RJ, Kuntz Iv C. Vertebral osteomyelitis. Neurosurg Clin N Am. 2006;17(3):339351 .

4. Berbari EF, Kanj SS, Kowalski TJ, et al. Infectious Diseases Society of America (IDSA) Clinical Practice Guidelines for the Diagnosis and Treatment of Native Vertebral Osteomyelitis in Adults. Clin Infect Dis. 2015;61(6):e26-e46.

5. Skaf GS, Domloj NT, Fehlings MG, et al. Pyogenic spondylodiscitis: an overview. J Infect Public Health. 2010;3(1):5-16.

6. Torda AJ, Gottlieb T, Bradbury R. Pyogenic vertebral osteomyelitis: analysis of 20 cases and review. Clin Infect Dis. 1995;20(2):320-328.

7. Grammatico L, Baron S, Rusch E, et al. Epidemiology of vertebral osteomyelitis (VO) in France: analysis of hospitaldischarge data 2002-2003. Epidemiol Infect. 2008;136(5):653660 .

8. Carragee EJ. Pyogenic vertebral osteomyelitis. J Bone Joint Surg Am. 1997;79(6):874-880.

9. Cottle L, Riordan T. Infectious spondylodiscitis. J Infect. 2008;56(6):401-412.

10. Karikari IO, Powers CJ, Reynolds RM, Mehta AI, Isaacs RE. Management of a spontaneous spinal epidural abscess: a single-center 10-year experience. Neurosurgery. 2009;65(5):919-923.

11. Darouiche R. Spinal epidural abscess. $N$ Engl J Med. 2006;355(19):2012-2020.

12. Zimmerer SME, Conen A, Müller AA, et al. Spinal epidural abscess: aetiology, predisponent factors and clinical outcomes in a 4-year prospective study. Eur Spine J. 2011;20(12):2228-2234.

13. Chenoweth CE, Bassin BJ, Hartley SE, et al.; Vertebral Osteomyelitis Guideline Team. Vertebral Osteomyelitis, Discitis, and Spinal Epidural Abscess in Adults. Ann Arbor, MI: University of Michigan; 2013. http://www.med.umich.edu/ 1info/FHP/practiceguides/vertebral/VO.pdf

14. Nickerson EK, Sinha R. Vertebral osteomyelitis in adults: an update. Br Med Bull. 2016;117(1):121-138.

15. Pola E, Logroscino CA, Gentiempo M, et al. Medical and surgical treatment of pyogenic spondylodiscitis. Eur Rev Med Pharmacol Sci. 2012;16(suppl 2):35-49. http://www.ncbi. nlm.nih.gov/pubmed/22655482.

16. Ghobrial GM, Beygi S, Viereck MJ, et al. Timing in the surgical evacuation of spinal epidural abscesses. Neurosurg Focus. 2014;37(August):E1.

17. Adogwa O, Karikari IO, Carr KR, et al. Spontaneous spinal epidural abscess in patients 50 years of age and older: a
15 -year institutional perspective and review of the literature. $J$ Neurosurg Spine. 2014;20(3):344-349.

18. Connor DE, Chittiboina P, Caldito G, Nanda A. Comparison of operative and nonoperative management of spinal epidural abscess: a retrospective review of clinical and laboratory predictors of neurological outcome. J Neurosurg Spine. 2013;19(1):119-127.

19. Deyo RA, Cherkin DC, Ciol MA. Adapting a clinical comorbidity index for use with ICD-9 administrative databases. $J$ Clin Epidemiol. 1992;45(10):613-619.

20. Ortman JM, Velkoff VA, Hogan H. An aging nation: the older population in the United States. Econ Stat Adm US Dep Commer. 2014;1964:1-28.

21. Miller JA, Achey RL, Derakhshan A, Lubelski D, Benzel EC, Mroz TE. Neurologic complications, reoperation, and clinical outcomes following surgery for vertebral osteomyelitis. Spine (Phila Pa 1976). 2015;41(4):1.

22. Kothari M, Shah K, Tikoo A, Nene A. Short to midterm term surgical outcome study with posterior only approach on tuberculous spondylodiscitis in an elderly population. Asian Spine J. 2016;10(2):258-266.

23. Arnold R, Rock C, Croft L, Gilliam BL, Morgan DJ. Factors associated with treatment failure in vertebral osteomyelitis requiring spinal instrumentation. Antimicrob Agents Chemother. 2014;58(2):880-884.

24. Mavrogenis AF, Igoumenou V, Tsiavos K, et al. When and how to operate on spondylodiscitis: a report of 13 patients. Eur J Orthop Surg Traumatol. 2016;26(1):31-40.

25. Akiyama $\mathrm{T}$, Chikuda $\mathrm{H}$, Yasunaga $\mathrm{H}$, Horiguchi $\mathrm{H}$, Fushimi K, Saita K. Incidence and risk factors for mortality of vertebral osteomyelitis: a retrospective analysis using the Japanese diagnosis procedure combination database. $B M J$ Open. 2013;3(3):1-6.

26. McHenry MC, Easley KA, Locker GA. Vertebral osteomyelitis: long-term outcome for 253 patients from 7 Cleveland-area hospitals. Clin Infect Dis. 2002;34(10):13421350.

27. Hutchinson C, Hanger C, Wilkinson T, Sainsbury R, Pithie A. Spontaneous spinal infections in older people. Intern Med J. 2009;39(12):845-848.

28. Matsui H, Hirano N, Sakaguchi Y. Vertebral osteomyelitis: an analysis of 38 surgically treated cases. Eur Spine J. 1998;7(1):50-54.

29. Pourtaheri S, Issa K, Stewart T, et al. Comparison of instrumented and noninstrumented surgical treatment of severe vertebral osteomyelitis. Orthopedics. 2016;39(3):e504-e508.

30. Hee HT, Majd ME, Holt RT, Pienkowski D. Better treatment of vertebral osteomyelitis using posterior stabilization and titanium mesh cages. $J$ Spinal Disord Tech. 2002;15(2):149-156; discussion 156.

31. Rayes M, Colen C, Bahgat D, et al. Safety of instrumentation in patients with spinal infection. $J$ Neurosurg Spine. 2010;12(6):647-659.

32. Cook NL, Hicks LS, O’Malley AJ, Keegan T, Guadagnoli E, Landon BE. Access to specialty care and medical services in community health centers. Health Aff. 2007;26(5):1459-1468.

33. Hasan O, Orav EJ, Hicks LS. Insurance status and hospital care for myocardial infarction, stroke, and pneumonia. J Hosp Med. 2010;5(8):452-459.

34. McDavid K, Tucker TC, Sloggett A. Cancer survival in 
Kentucky and health insurance coverage. Arch Intern Med. 2003;163(18):2135-2144.

35. Bradley CJ. Disparities in cancer diagnosis and survival. Cancer. 2001;91(1):178-188.

36. Roetzheim RG, Pal N, Gonzalez EC, Ferrante JM, Van Durme DJ, Krischer JP. Effects of health insurance and race on colorectal cancer treatments and outcomes. Am J Public Health. 2000;90(11):1746-1754.

37. Furlan JC, Craven BC, Massicotte EM, Fehlings MG. Early versus delayed surgical decompression of spinal cord after traumatic cervical spinal cord injury: a cost-utility analysis. World Neurosurg. 2016;88:166-174.

38. Mogal H, Vermilion SA, Dodson R, et al. Modified frailty index predicts morbidity and mortality after pancreaticoduodenectomy. Ann Surg Oncol. 2017;24(6):1714-1721.

39. Klabunde CN, Potosky AL, Legler JM, Warren JL. Development of a comorbidity index using physician claims data. J Clin Epidemiol. 2000;53(12):1258-1267.

Disclosures and COI: The seventh author received personal consulting fees for Spinewave and Medicrea. The eighth author received personal consulting fees for DePuy Synthes. The first through sixth authors and the ninth author report no conflicts of interest. Each institution obtained approval from its local institutional review board, in which this study was deemed exempt due to the deidentified nature of the data. No sources of funding.

Corresponding Author: Peter G. Passias, MD, New York Spine Institute, NYU Medical Center, Hospital for Joint Diseases, Department of Orthopaedic Surgery, 301 East 17th Street, New York, NY 10003. Phone: (516) 357-8777; Fax: (516) 357- 0087; Email: address: pgpassias@yahoo.com.

Published 21 December 2018

This manuscript is generously published free of charge by ISASS, the International Society for the Advancement of Spine Surgery. Copyright @ 2018 ISASS. To see more or order reprints or permissions, see http://ijssurgery.com.

\section{APPENDIX}

Surgical Procedure ICD-9 Codes: 03.09, 03.53, 03.99, 77.49, 77.70, 80.51, 81.00, 81.01, 81.02, $82.03,81.04,81.05,81.06,81.07,81.08,81.09$, $81.62,81.63,81.64,84.51,84.52,81.30,81.32$, $81.33,81.34,81.35,81.36,81.37,81.38$

Vertebral Osteomyelitis ICD-9 Codes: 730.08, $730.28,722.90,722.91,722.93$ and 722.93

Incomplete Neurologic Deficit ICD-9 Codes: 344.0, 344.00, 344.02, 344.04, 344.1, 344.2, 344.30, 344.31,
$344.32,344.40,344.41344 .42,344.5,722.71722 .73$, 722.72, 724.4, 723.4, 721.1, 721.42, 721.41, 336.8

Complete Neurologic Deficit ICD-9 Codes: 344.01, 344.03

Diabetes: 29002901029011290122901329020 2902129032904029041290422904329082909

Chronic Pulmonary Disease: 4904910491149120 $\begin{array}{llllllll}49121 & 49122 & 4918 & 4919 & 4920 & 4928 & 49300 & 49301\end{array}$ 4930249310493114931249320493214932249381 4938249390493914939249404941495049514952 4953495449554956495749584959496500501 5025035045055064

Myocardial Infarction: 4100041004101041011 4101241020410214102241030410314103241040 4104041041410424105041051410524106041061 4106241070410724108041081410824109041091 41092412

Rheumatological Conditions:7100710171047140 7141714271481725

Renal: 5820582158225824582815828958295830 5831583258345836583758515852585358535854 5855585658595865880588158881588895889

Congestive Heart Failure: 428042814282042821 4282242823428304283142833428404284142842 428434289

Diabetes with Complications: 250402504125042 25043250502505125052250532506025061250062 25063

PVD: 44394410044101441024410344114412 44134414441544164417441978543848738482

Cancer: 14001401140314041405140614081409 1410141114121413141414151416141814191420 1421142214281429143014311438143914401441 1448144914501451145214531454145514561458 1459146014614162146314641465146614671468 1469147014711472147314781479148014811482 1483148814891490149114981499150015011502 1503150415051508150915101511151215131514 1515151615181519152015211522152315281529 1530153115321533153415351536153715381539 1540154115421543154815501551155215601561 1562156815691570157115721573157415891579 1580158815891590189115981599160016011602 1603160416051608160916101611161216131618 1619162016221623162416251628162916301631 1638163916401641164216431648164916501658 1659170117021703170417051706170717081709 
1710171217131714171517161717171817191720 1721172217231724172517261727172817291740 1741174217431744174517461748174917501759 176017611762176317641765176817691791800 180118081809181182018211828183018321833 1834183518381839184018411842184318441848 184918518601869187118721873187418751876 1877187818791880188118821883188418851886 1887188818891890189118921893189418981899 1900190119021903190419051906190719081909 1910191119121913191419151916191719181919 192019211922192319281929193194019411943 1944194519461948194919501951195219531954 1955195820000200012000220003200042005 2000620007200082001020011200122001320014 2001520016200172001820020200212002220023 2002420025200262002720028200302003120032 2003320034200352003620037200382004020041 2004220043200442004520046200472004820050 2005120052200232002420055200562005720058 2006020061200622006320064200652006620067 2007020071200722007320074200752007620077 2007820080200812008220083200842008520086 2008720088201002010120102201032010420105 2010620107201082011020111201122011320114 2011520116201172011820120201212012220123 2012420125201262012720128201302013120132 2013320134201352013620137201382014020141 2014220143201442014520146201472014820150 2015120152201532015420155201562015720158 2016020161201622016320164201652016620167 2016820170201712017220173201742017520176 2017720178201902019120192201932019420195 2019620197201982020020201202022020320204 2020520206202072020820210202112021220213 2021420215202162021720218202202022120222 2022320224202252022620227202282023020231 2023220233202342023520236202372023820240 2024120242202432024420245202462024720248 2025020251202522025320254202552025620257 2025820260202612026220263202642026520266 2026720268202702027120272202732027420275 2027620277202782028020281202822028320284 2028520286202872028820290202912029220293 2029420295202962029720298203002030120302 2031020311203122038020381203822040020401 2040220410204112041220420204212042220480 2048120490204912094220500502012050220510 2051120512205202052120522205302053120532
2058020581205822059020591205922060020601 2060220610206112061220620206212062220680 2068120682206902069120692207002070120702 2071020711207122072020721207222078020781 2078220800208012080220810208112081220821 20822208802088120882208902089120892

Any Stroke: 4304314320432143294330043301 4331143320433214333043331433804338143390 433914340043401434104341143490434914350 435143524353435843594364370437143724373 43744375437643774378437943804381043811 4381243813438144381943820438214382243830 4383143832438404384143842438504385143852 43853438643874388143882438834388443885 438894389

Peptic Ulcer Disease: 53100531015311053111 5312053121531305313153140531415315053151 5316053161531705317153190531915320053201 5321053211532205322153230532315324053241 5325053251532605326153270532715329053291 5330053301533105332053321533305333153340 5334153350533515336053361533705337153390 5339153400534015341053411534205342153430 5343153440534415345053451534605346153470 534715349053491

Liver Disease: 45604561456204652157225723 57245728

Metastasis: 1960196119621963196519661968 1969197019711972197319741975197619771978

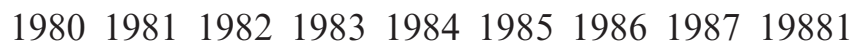
198821988919901991

AIDS: 42420421422429430431432433439440 449

Severe Liver Disease: 4560456145620465215722 572357245728

Dementia: 29002901029011290122901329020 2902129032904029041290422904329082909

Anemia: 2851

Device: 9960099649964099641996429964399644 99645996469964799649

Infection: 99859985199859

Sepsis: 9959199592 